${ }^{1}$ Centro Especializado de Genética (CEGEN), Facultad de Ciencias Naturales y Exactas, Universidad Autónoma de Chiriquí (UNACHI), ciudad de David, provincia de Chiriquí, República de Panamá. ${ }^{2}$ Centro Gendiagnostik, S.A., ciudad de David, provincia de Chiriquí, República de Panamá. aLicenciada en Biología. bGeneticista clínica molecular, M.Sc., Dr.rer.nat.

Fuente de apoyo financiero:

Este artículo fue financiado por el Centro Gendiagnostik, el Sistema Nacional de Investigación (SNI), la Secretaría Nacional de Ciencia, Tecnología e Innovación (SENACYT/ APY-NI10-002 A) y la Vicerrectoría de Investigación y Posgrado de la UNACHI (VIP 184-CN-01-A092-022011).

Recibido el 22 de noviembre de 2014, aceptado el 9 de julio de

Correspondencia a: Dra. Oriana Batista Centro Gendiagnostik, Avenida Domingo Díaz y Calle Central. Ciudad de David, provincia de Chiriquí, República de Panamá. Apdo. postal: 0426-01262. Telefax: (507)-7742871 jbbo@gmx.net

\section{Neurofibromatosis tipo 1 (NF1) y su diagnóstico molecular como estrategia del diagnóstico diferencial y a edades tempranas}

\author{
MARTHA GÓMEZ ${ }^{1, a}$, ORIANA BATISTA ${ }^{1,2, b}$
}

\section{Molecular diagnosis as a strategy for differential diagnosis and at early ages of neurofibromatosis type 1 (NF1)}

Neurofibromatosis type 1 (NF1), is a haploinsufficient and multisystemic disease, caused by inherited or sporadic mutations in the NF1 gene. Its incidence is one in 2,500 to 3,000 individuals, it has an autosomal dominant pattern of inheritance, high clinical variability, complete penetrance and age-dependent complications. Neurofibromin is the product of the NF1 gene and is believed to act as a tumor suppressor since the loss of its function has been associated with benign and malignant tumors in neural crest-derived tissues. Only two correlations between clinical phenotype and mutant alleles in the NF1 gene have been observed. The established criteria for disease diagnosis are very efficient in adults and children older than 3 years of age, but not for children under this age. Mutational analysis is therefore recommended to confirm the disease in young children with a negative family history. A pathogenic mutation in the NF1 should be added to the list of diagnostic criteria. Mutational analysis is also recommended for differential diagnosis and for prenatal or pre-implantation genetic diagnosis, taking into consideration the family history and the type of method to be applied. Molecular studies of this disease using different complimentary molecular techniques and bioinformatics tools have characterized NF1 gene mutations at both the DNA and mRNA levels, increasing the mutational spectrum. Consequently, about 1,289 defects have been reported to date, mainly nonsense/missense mutations, deletions and splice site defects.

(Rev Med Chile 2015; 143: 1320-1330)

Key words: Child; Computational Biology; DNA Mutational Analysis; Neurofibromatosis 1; Neurofibromin 1.
L a Neurofibromatosis tipo 1 (NF1; MIM \#162200), es un desorden con patrón de herencia autosómico dominante e incidencia de 1 en 2.500 a 3.000 individuos $^{1}$. Es una enfermedad multisistémica ocasionada por mutaciones heterogéneas en el gen $N F 1$, las cuales son esporádicas en, aproximadamente, $50 \%$ de los casos ${ }^{2}$. NF1 fue identificado y su proteína ca- racterizada, por Cawthon y cols. y Wallace y cols., respectivamente, en 1990. La secuencia del ADNc fue descrita por Gutmann y Collins y Viskochil y cols., en $1993^{3}$.

A pesar de que, aproximadamente, $1289 \mathrm{mu}-$ taciones han sido reportadas (HGMD-Human Gene Mutation Database), pocas son las relaciones genotipo-fenotipo, descritas. 
NF1 se caracteriza por extrema variabilidad clínica entre individuos afectados no relacionados, entre miembros afectados de una familia, y en una persona en diferentes etapas de su vida. Adicionalmente, la frecuencia de complicaciones se incrementa con la edad ${ }^{3}$.

El diagnóstico clínico de los pacientes con NF1, se basa en los criterios establecidos en la conferencia para el desarrollo de consensos del Instituto Nacional de Salud (siglas en inglés NIH), en 19884, los cuales toman en consideración la condición de afectación de piel, huesos y sistema nervioso, principalmente. Aunque estos criterios resultan ser altamente específicos y sensitivos en adultos ${ }^{5}$, no se presentan en algunos niños menores de 8 años ${ }^{6}$. Para este último grupo, el diagnóstico molecular sería útil, pues aproximadamente, la mitad de aquellos que están afectados y no presentan antecedentes familiares, reúnen los criterios para el diagnóstico al año de edad, y la mayoría de ellos, cumplirán con éstos a los 8 años.

Aunque en algunos laboratorios el diagnóstico genético de NF1 es rutinario, algunos factores tales como su gran tamaño, la carencia de sitios específicos de mutación y la presencia de pseudogenes, hacen laboriosa la identificación de mutaciones en NF1. A continuación, se presenta una revisión de algunos aspectos moleculares de la NF1, sus características genéticas, manifestaciones, criterios para el diagnóstico clínico, y algunos estudios mutacionales del gen. Adicionalmente, se muestra la utilidad del análisis mutacional como aporte al diagnóstico en niños menores, al diagnóstico diferencial de la enfermedad y al pre-natal y/o preimplantacional.

\section{El gen NF1 codifica la proteína neurofibromina, asociada con la supresión tumoral}

El gen NF1 se localiza en el cromosoma $17 q 11.2^{7-9}$, tiene un tamaño genómico de 282,751 pb, 60 exones, y codifica al menos tres transcritos solapados ${ }^{10}$. Cuatro exones alternativamente empalmados ${ }^{11}$ y pseudogenes en los cromosomas $2,14,15,18,21$ y $22^{3}$ han sido descritos. Adicionalmente, en el intrón 27 se localizan los genes EVI2A, EVI2B (ecotropic viral integration site $2 A y$ 2B) y OMGP (oligodendrocyte-myelin glycoprotein) que se transcriben en dirección opuesta al $N F 1^{12-14}$.

La transcripción de NF1 ocurre en dirección centrómero-telómero, y genera un ARNm de 11 a $13 \mathrm{~kb}$, con un marco de lectura abierto de, aproximadamente, $9 \mathrm{~kb}$. Su producto proteico, neurofibromina, tiene $280 \mathrm{kDa}$ y expresión ubicua ${ }^{11}$ en fibroblastos de piel, cerebro, bazo, pulmón, músculo, neuroblastoma, neurofibroma, células de neurofibrosarcoma NF1, carcinoma de colon y cáncer de mama, entre otros ${ }^{8,15,16}$.

La función de los 3 dominios que forman la proteína $^{17-19}$ se comprende parcialmente. El dominio GRD (GAP-related domain) de, aproximadamente, 360 aminoácidos, ubicado en la región central de la neurofibromina y determinado por los exones 20 a 27a, muestra homología estructural y funcional con la familia de proteínas activadoras de GTPasa (GAP). Este dominio interactúa con la proteína ras-GTP y promueve la conversión de ras-GTP a su forma inactiva ras-GDP regulando negativamente la vía de señalización p21 ras $^{17,20,21}$. Consecuentemente, a la neurofibromina se le considera un supresor tumoral ${ }^{22}$, pues la reducción de su producción (en células haploinsuficientes) o la completa pérdida de ésta, conlleva la activación de la proteína ras, que a su vez regula una cascada de vías de señalización posteriores, entre ellas, las proteínas quinasas activadas por mitógenos (MAPK), fosfoinositol 3-quinasas (P13K), proteína quinasa $\mathrm{B}(\mathrm{PKB})$ y proteína quinasa diana de rapamicina en células de mamífero $(\mathrm{mTOR})^{3}$. La activación de estas vías, ocasiona una variedad de efectos celulares que, generalmente, estimulan la supervivencia y proliferación celular ${ }^{23}$ (Figura 1).

La pérdida de la función de esta proteína, se asocia con tumores benignos y malignos encontrados en tejidos derivados de la cresta neural ${ }^{24}$. Así, generalmente, en ausencia o reducción drástica de la neurofibromina, se pueden encontrar niveles elevados de ras-GTP en tumores de los nervios periféricos de pacientes con $\mathrm{NF}^{25}$. El aumento del riesgo de tumores malignos en NF1, es compatible con la pérdida de función de la neurofibromina, como regulador negativo del oncogén $\operatorname{ras}^{21}$.

Los otros dominios potenciales funcionales en la neurofibromina son: un dominio rico en residuos de cisteína y serina (CSRD) entre el exón 11 y 17 que codifica varios sitios de reconocimiento para proteína quinasa dependiente de AMP cícli$\mathrm{Co}^{18}$, y el dominio NF1-Sec-PH relacionado con la unión a fosfolípidos ${ }^{19}$. Interacciones directas o indirectas entre la neurofibromina y otras proteínas, también han sido documentadas, aunque no se conoce su significado biológico ${ }^{26}$. 


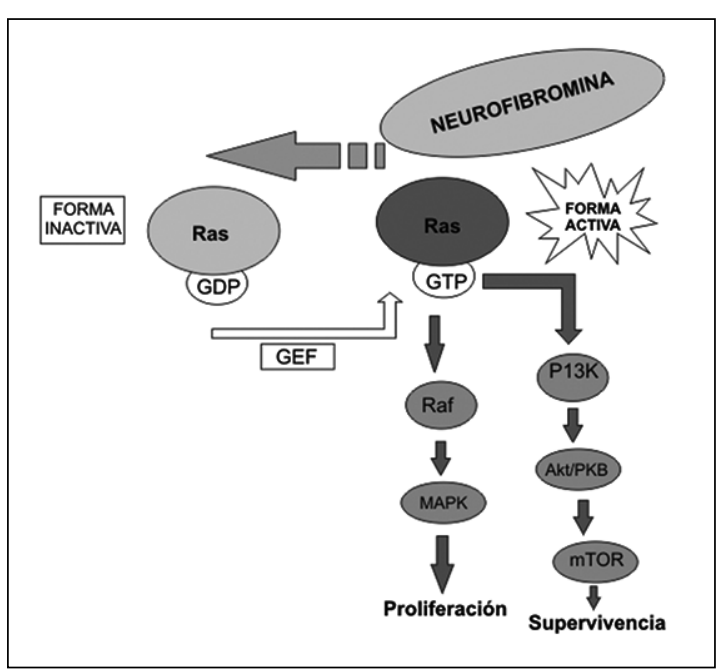

Figura 1. La neurofibromina actúa como regulador negativo de la vía de señalización Ras, promoviendo su forma inactiva Ras-GDP. La neurofibromina es un tipo de proteína activadora de la actividad GTPasa (GAP) que se une a la forma activa de la proteína ras (ras-GTP) y la inactiva (ras-GDP) y, consecuentemente, no hay proliferación ni supervivencia celular alterada. Los factores de intercambio de nucleótidos de guanina (GEF) realizan el paso contrario, reactivando a Ras al promover el intercambio de GDP a GTP. La pérdida de la función de la neurofibromina, en individuos NF1 heterocigotos y homocigotos evita la conversión de Ras-GTP a Ras-GDP, y esto a su vez, desencadena cascadas de señalización posteriores con efectos de supervivencia y proliferación celular, entre otros (ver texto para información detallada). Abreviaturas: Ras-GDP, forma inactiva de la proteína Ras unida a guanosín difosfato. Ras-GTP: forma activa de la proteína Ras unida a guanosín trifosfato. Raf: serina-treonina proteína cinasa. MAPK: proteínas cinasas activadas por mitógenos. P13K: fosfoinositol 3-cinasas. Akt/PKB: proteína cinasa B. mTOR: proteína cinasa diana de rapamicina en células de mamífero.

\section{La NF1 se hereda de forma autosómica dominante, su fenotipo es variable $y$ completamente penetrante}

La pérdida de función de un gen es causada por mutaciones que interrumpen su capacidad codificante $^{27}$. NF1 exhibe un patrón de herencia autosómico dominante, los individuos afectados tienen un genotipo heterocigoto y la descendencia de éstos tiene $50 \%$ de riesgo de heredar el gen alterado ${ }^{28}$. La mutación en los individuos heterocigotos causa haploinsuficiencia, y por ello, la única copia funcional no produce suficiente neurofibromina para asegurar un desarrollo y crecimiento celular normal ${ }^{26}$. No existen individuos homocigotos ${ }^{26}$ y los ratones homocigotos $\left({\mathrm{N} f 1^{-/}}^{-}\right)$ mueren en el útero, lo cual indica la importancia vital de la neurofibromina ${ }^{29,30}$. La penetrancia de NF1 es $100 \%{ }^{26}$ y se completa, esencialmente, entre los cinco y ocho años de edad ${ }^{6,40}$.

NF1 tiene una de las tasas de mutación más altas y las mutaciones esporádicas ocurren casi en la mitad de los pacientes afectados ${ }^{31,32}$. Las mutaciones esporádicas en NF1 de la línea germinal, están sesgadas hacia el sexo. De éstas más de $80 \%$ tienen un origen paterno ${ }^{33}$. Otras mutaciones, llamadas microdeleciones del locus, tienen, en su mayoría, un origen materno ${ }^{34}$, abarcan, aproximadamente, $1,5 \mathrm{Mb}$ e incluyen $N F 1$ y regiones flanqueadoras ${ }^{35}$.

Gran variabilidad fenotípica entre pacientes con NF1, se aprecia intra e inter-familiarmente, aún cuando se presentan mutaciones idénticas en el gen ${ }^{36}$. Entre familias de Corea con la misma mutación, se observó que unos miembros manifestaron patologías malignas y otros menos severas. Intra-familiarmente, aunque madre e hija presentaron una deleción, del exón 2 al 47 (c.61-8315del), la primera, de 29 años, desarrolló sólo manifestaciones cutáneas, y la segunda, de 6,2 años, glioma cerebral, pseudoartrosis severa de tibia y escoliosis ${ }^{37}$.

Esta expresividad variable, podría deberse al efecto de genes modificadores o mutaciones somáticas secundarias dentro de los tejidos tumorales ${ }^{38,39}$. Adicionalmente, variantes de un gen podrían afectar la estructura y función de la proteína, y ocasionarían, así, fenotipos variables ${ }^{40}$.

Sólo dos correlaciones genotipo-fenotipo, las cuales, también, son ejemplos de la variabilidad fenotípica de NF1 han sido observadas: 1) la deleción completa del gen, microdeleciones $N F 1$, caracterizada por la aparición temprana y numerosa de neurofibromas cutáneos, con mayor frecuencia y la severidad de anormalidades cognitivas, malformaciones, tumoración y riesgo aumentado de malignización ${ }^{41,42}$; y 2) la deleción específica de tres pb en el exón 17, c.2970-2972 delAAT (p.990delM), asociada con pigmentación, nódulos de Lisch y ausencia de neurofibromas cutáneos o neurofibromas plexiformes ${ }^{40}$.

\section{Las manifestaciones clínicas como criterios para el diagnóstico}

El diagnóstico de pacientes con NF1 se basa, primordialmente, en los criterios $\mathrm{NIH}^{4}$ (Tabla 1), 
Tabla 1. Criterios para el diagnóstico clínico de la Neurofibromatosis tipo 1

- Seis o más manchas café con leche mayores de $5 \mathrm{~mm}$ en pacientes prepuberales y mayores de $15 \mathrm{~mm}$ en pacientes postpuberales

- Dos o más neurofibromas de cualquier tipo o un neurofibroma plexiforme

- Signo de Crowe (efélides axilares o inguinales)

- Glioma de nervio óptico

- Dos o más nódulos de Lisch (hamartomas de iris)

- Lesiones óseas típicas (displasia del esfenoides, displasia o adelgazamiento cortical de huesos largos con o sin pseudoartrosis)

- Antecedentes de neurofibromatosis tipo I en padres o hermanos

Si 2 o más de estos criterios son encontrados en un individuo se realiza el diagnóstico de NF1.

pero también en otras manifestaciones, con frecuencia media y ocasional, abajo mencionadas ${ }^{5}$. Éste requiere de un examen clínico cuidadoso del paciente, sus padres, hermanos y una historia familiar detallada, que incluya información clínica y en ocasiones exámenes complementarios ${ }^{43}$.

Las manifestaciones clínicas más frecuentes en pacientes con NF1 son las alteraciones en las manifestaciones de la piel (más de 99\%), neurofibromas cutáneos (más de 99\%) [5 en superíndice] , nódulos de Lisch (de 90-95\%) y efélides $(85 \%)^{5,40}$. Otras complicaciones en diversos sistemas podrían presentarse. Las discapacidades de aprendizaje, desarrollo de anormalidades esqueléticas, enfermedades vasculares, tumores del sistema nervioso central o malignos de la vaina de los nervios periféricos se presentan con frecuencia ${ }^{3}$. Algunas complicaciones raras, que afectan a menos de $5 \%$ de los pacientes incluyen epilepsia, hidrocefalia, problemas cardiovasculares y escoliosis distrófica ${ }^{44}$.

Los tumores del sistema nervioso central y los neurofibromas podrían resultar en importante morbilidad y mortalidad ${ }^{45}$ y reducir la esperanza media de vida de las personas con NF1 en, aproximadamente, 15 años. Las causas más importantes de muerte temprana son las manifestaciones malignas y enfermedades vasculares ${ }^{3,36,46}$. Individuos entre 20-35 años tienen 8-13\% de riesgo de desarrollar tumores malignos de la vaina de los nervios periféricos, predominantemente ${ }^{47}$, los cuales son difíciles de detectar, fácilmente metastásicos y a menudo de mal pronóstico ${ }^{48}$.

Fenotipos leves de NF1 son causados por mosaicismo originado por mutaciones después de la fertilización ${ }^{26}$ y se clasifican como segmentario, generalizado, o gonadal ${ }^{49}$. El generalizado es clínicamente similar a la NF1 clásica, pero se presenta poca o ninguna evidencia de la enfermedad al examinar los leucocitos sanguíneos para detectar mutaciones. El segmentario ocasiona regiones con alteraciones pigmentarias, crecimientos tumorales, o ambos, limitados a una o más regiones del cuerpo ${ }^{50}$. El gonadal, causa mutaciones en las gónadas, y puede deducirse cuando dos o más hijos de padres no afectados desarrollan la enfermedad ${ }^{51}$.

\section{Utilidad del análisis mutacional para el diagnóstico de la NF1 en niños menores, en el diagnóstico diferencial y en el diagnóstico prenatal o preimplantacional}

El análisis mutacional es, especialmente, útil en niños pequeños con historia familiar negativa y que llenan parcialmente los criterios de diagnóstico NIH, en niños con presentaciones atípicas ${ }^{3}$, en el diagnóstico diferencial o en el diagnóstico genético prenatal o preimplantacional ${ }^{5,50}$.

Aunque los criterios de diagnóstico NIH son útiles para la detección de NF1, algunos de éstos se manifiestan a una edad superior a los 3 años y están presentes al nacimiento sólo en algunos pacientes ${ }^{5}$. Por ello, el diagnóstico definitivo, usando dichos criterios puede ser realizado, para la mayoría entre los cuatro y cinco años ${ }^{28,40}$. Aproximadamente $46 \%$ de los casos esporádicos de NF1, no cumplen con dichos criterios a la edad de un año ${ }^{6}$.

Seis o más manchas café con leche se presentan desde el nacimiento, en más de $99 \%$ de los casos con NF1 y este criterio es el más frecuente ${ }^{5,6}$ y altamente sugestivo de la enfermedad ${ }^{52}$. Sin embargo, aproximadamente, $1 \%$ de los niños que manifestarán la enfermedad a edades superiores, podrían presentar un número inferior a seis manchas, lo cual no ayudaría en el diagnóstico clínico, pues menos de $1 \%$ de los niños menores de 5 años sin NF1, presentan más de dos manchas ${ }^{52}$. Otros infantes, cuyos padres no muestran signos de NF1, muestran manchas café con leche, sin otras manifestaciones ${ }^{3}$. 
La prevalencia de la NF1 en Irlanda del Norte, en menores de 16 años ${ }^{53}$, fue 17,6 por cada 100.000 ( 1 en 5.681) niños y éstos desarrollaron numerosas complicaciones. Las más frecuentes, desde el nacimiento, incluyen dificultades de aprendizaje (30-60\%), neurofibromas plexiformes (30-50\%), escoliosis $(10 \%)^{5}$ y glioma del nervio óptico (15$20 \%)^{5,36}$. Estos últimos, se presentan antes de los seis años, pero podrían volverse sintomáticos en la infancia tardía o la adultez y causar ceguera ${ }^{28}$.

Un análisis mutacional exhaustivo permitiría identificar mutaciones en un poco más de $92 \%$ de pacientes que cumplen con los criterios $\mathrm{NIH}^{54,55}$. Este análisis, además, de permitir la confirmación del diagnóstico de NF1, facilitaría la identificación de las mutaciones en el afectado, útiles para diagnósticos pre-natales y pre-implantacionales de su futura descendencia debido a que sólo $50 \%$ de ésta tiene la probabilidad de tener la enfermedad. Mientras que el resultado del diagnóstico pre-natal podría sugerir a la pareja la interrupción terapéutica del embarazo, el diagnóstico pre-implantacional podría evitarlo ${ }^{56}$.

Los estudios mutacionales apoyarían, también, el diagnóstico diferencial de la NF1 respecto a síndromes como Noonan, LEOPARD, síndrome cardiofaciocutáneo, Costello, Legius, entre otros $^{5,26}$. Estas condiciones comparten un grado variable de dificultad de aprendizaje, defectos cardiacos, dimorfismo facial, baja estatura, macrocefalia y anormalidades de la piel ${ }^{57}$. Por ejemplo, pacientes con fenotipo leve, quienes cumplen con los criterios para NF1, podrían padecer el síndrome Legius, caracterizado por mutación en SPRED $1^{58}$. Aproximadamente, 1 a $2 \%$ de pacientes que cumplen con los criterios de diagnóstico NF1, presentan mutaciones en SPRED $1^{59}$.

En resumen, todos los pacientes con mutación en NF1 manifestarán la enfermedad debido a que la penetrancia de $\mathrm{NF} 1$ es $100 \%{ }^{26}$, pero no todos cumplirán con los criterios $\mathrm{NIH}$ antes de los cuatro o cinco años. Algunos pacientes que llenan estos criterios y exhiben fenotipos leves podrían presentar otros síndromes. Por estas razones, los estudios mutacionales pueden proveer información oportuna y una confirmación del diagnóstico clínico para un manejo personalizado del paciente. Adicionalmente, la información generada, mediante estos análisis, sería útil para los diagnósticos pre-natales, pre-implatacionales y las asesorías genéticas. Así, la idea de modificar ${ }^{6}$ e incluir la presencia de una mutación patogénica a los criterios $\mathrm{NIH}^{50,57}$ para facilitar el diagnóstico a edades muy tempranas es razonable. El aumento en la cantidad de estudios de diagnóstico molecular y en el número de mutaciones, también, demuestra la utilidad de los mismos para confirmar el diagnóstico clínico.

\section{Panorama del análisis mutacional en NF1}

La determinación de mutaciones en NF1 más que compleja es laboriosa, debido a numerosos exones codificantes, heterogeneidad mutacional, tamaño del gen y la ausencia de "puntos calien-

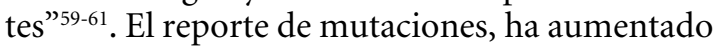
desde 1992, cuando se creó el International NF1 Genetic Analysis Consortium con la finalidad de detectar las mutaciones y organizar la información creciente en bases de datos ${ }^{18}$. Así, en el consorcio se reportaron más de 100 mutaciones en la línea germinal en $1996^{62}$ y 240 en $1997^{63}$. HGMD ( $\mathrm{Hu}$ man Gene Mutation Database), reportó más de 400 mutaciones en $2004^{64}$ y más de 1.289 , que incluyen mutaciones sin sentido, con sentido equivocado, errores de empalme; deleciones, inserciones e indels pequeñas; deleciones e inserciones gruesas; complejos y repeticiones en mayo de 2015.

Diversas técnicas moleculares utilizadas en estudios provenientes de países como Alemania ${ }^{18}$, Italia $^{60,65,66}$, España ${ }^{22,61}$, Brasil $^{64}, \mathrm{Cuba}^{67}$, Corea $^{37} \mathrm{y}$ Estados Unidos de Norteamérica ${ }^{69}$, ofrecen un amplio rango de sensibilidad para la detección de mutaciones en NF1. Las mismas, se pueden identificar en aproximadamente, $85-95 \%$ de los casos, utilizando una combinación de técnicas moleculares $^{5}$. El porcentaje de sensibilidad mayor, $95 \%$, se obtuvo al utilizar las técnicas combinadas MLPA, RT-PCR, DHPL y secuenciación ${ }^{22}$ (Tabla 2).

Entre las métodos utilizados en los estudios anteriores figuran los de barrido tales como: SSCP, TGGE, DHPLC y PTT y otros a saber: secuenciación, qPCR, RT-PCR, MLPA y FISH (Tabla 2). Algunos laboratorios utilizan los métodos "de barrido", para minimizar los costos, pues aunque éstos no identifican el tipo de mutación, permiten descartar los exones normales, de los exones mutados en aquellos genes con heterogeneidad alélica fuerte, es decir, con posibilidad de mutaciones a lo largo de todo el gen. La detección exacta de la mutación debe realizarse, posteriormente, 


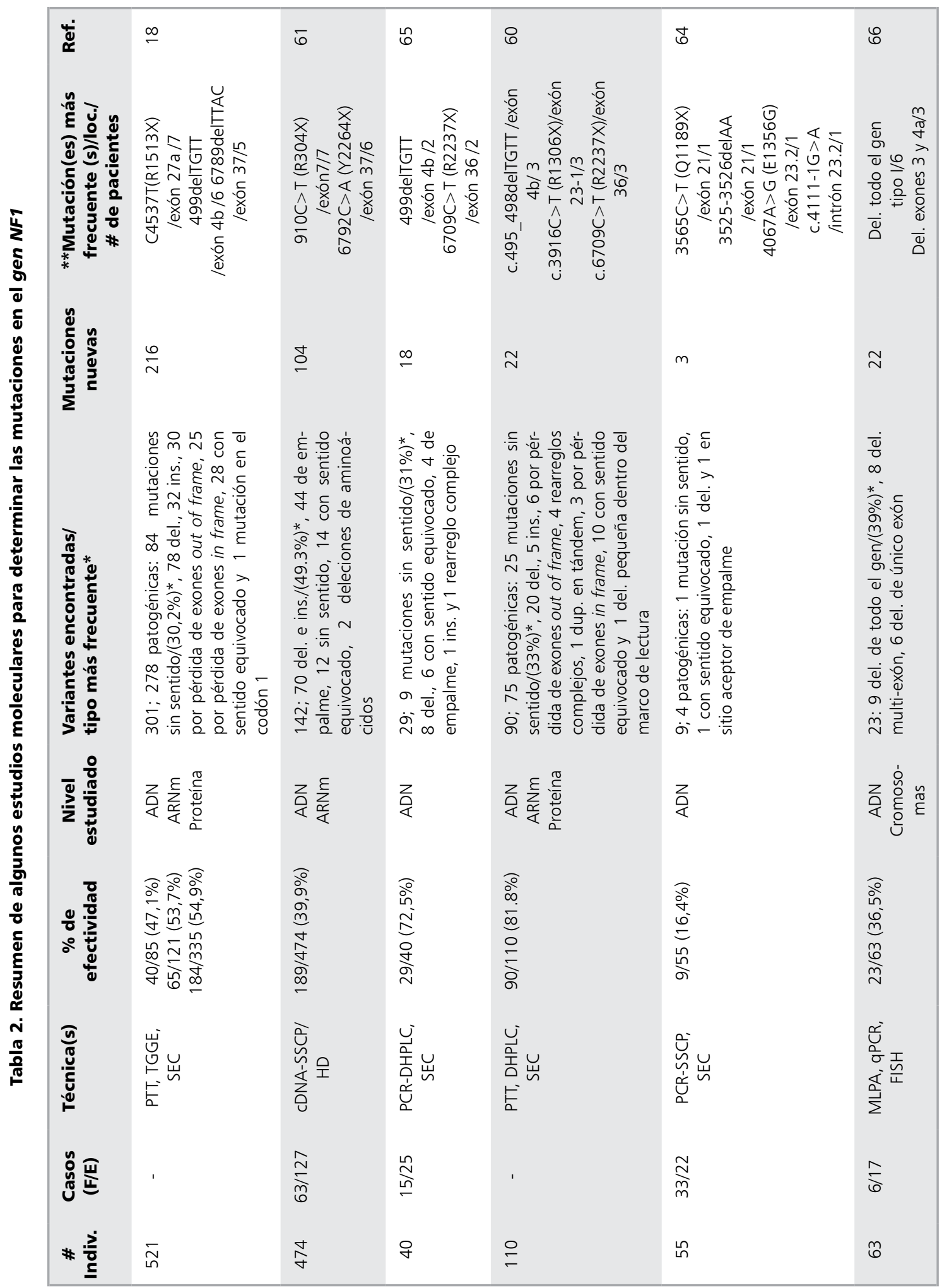




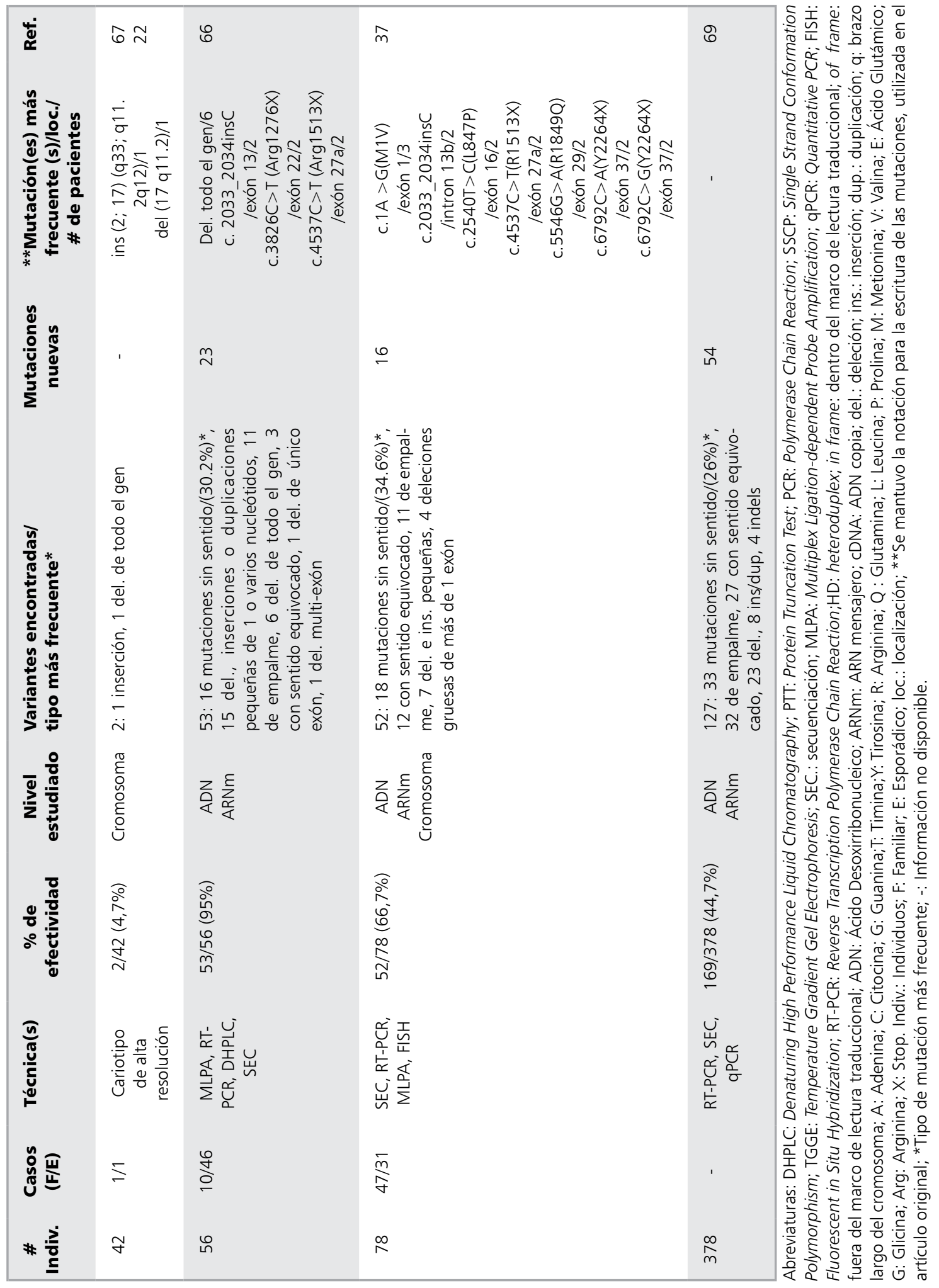


mediante secuenciación ${ }^{68}$, técnica que utiliza, frecuentemente, $\mathrm{ADN}$ genómico o ADN copia obtenido por RT-PCR, para detectar mutaciones puntuales que oscilan entre una base y algunas decenas de bases. Otros laboratorios con mayor facilidad económica o que conocen la mutación puntual existente en la familia utilizan la secuenciación como primera alternativa y esta práctica ha aumentado debido a la disminución de los costos asociados a la secuenciación en los últimos años. El MLPA, FISH, cariotipos, y métodos de análisis de múltiples polimorfismos de un solo nucleótido (SNPs) se utilizan para identificar mutaciones gruesas, es decir aquellas que involucran segmentos grandes incluso varios genes, y por lo tanto, permiten la detección de la deleción completa del gen, deleción de exones, duplicaciones y rearreglos cromosómicos.

El porcentaje de detección de mutaciones varía de acuerdo con los protocolos de análisis utilizados. Mientras que el protocolo de varios pasos, basado tanto en la secuencia del ADNc, ADN genómico, así como la deleción completa de NF1 identifica más de $95 \%$ de los casos, el de la secuencia genómica, únicamente, identifica, aproximadamente, $61 \%$. El análisis de deleciones y duplicaciones, determina aproximadamente, 5\% y el citogenético menos de $1 \%{ }^{28}$.

Abundantes mutaciones (Tabla 2) fueron localizadas uniformemente en NF1, desde el exón $1 \mathrm{al} 47^{18,37}$ y decayeron en densidad en los últimos exones $^{22}$. El tipo de mutación más frecuente fue la sin sentido, producida por la introducción de un codón de parada prematuro, que a menudo altera el empalme del $\mathrm{ARNm}^{28}$ y origina formas truncadas de neurofibromina, observadas en $80 \%^{18}, 77 \%{ }^{61}, 81 \%^{60}, 76,9 \%{ }^{37}$ y $72 \%{ }^{22}$ de los casos.

Aunque las mutaciones sin sentido causan la terminación prematura de la proteína, las deleciones e inserciones pequeñas generan transcritos con corrimiento de lectura que, también, podrían producir proteínas truncadas. Igualmente, las mutaciones en sitios de empalme y deleciones gruesas en el gen, pueden generar proteínas acortadas ${ }^{37}$.

Mutaciones con sentido equivocado, las cuales producen sustitución de aminoácidos, en sitios conservados dentro de la proteína ${ }^{18,37,69}$, también, se reportaron y por la ubicación de su ocurrencia podrían resultar en un cambio funcional ${ }^{69}$. Deleciones completas del gen, se han encontrado, generalmente, entre $4-5 \%$ de los individuos con
$\mathrm{NF} 1^{70}$. Otros estudios mostraron porcentajes similares $4,5 \%{ }^{66}$, o aproximados $11 \%{ }^{22}, 1,59 \%{ }^{69}$.

Herramientas y bases de datos bioinformáticas han sido utilizadas para caracterizar mutaciones en NF1: para el modelado molecular ${ }^{60,64}$, diseño de oligonucleótido ${ }^{64,65}$ y determinación de mutaciones $^{22,40,69}$. En la actualidad se utilizan herramientas computacionales para determinar los mecanismos de errores de empalme, la patogenicidad de las mutaciones y el alineamiento de proteínas ${ }^{69}$.

En resumen, la existencia de varios métodos moleculares así como el cúmulo de herramientas bioinformáticas aunadas a una estrategia metodológica bien planificada para la identificación de mutaciones en NF1, permitirá su identificación sin mayor dificultad. En este contexto, se sugiere considerar los siguientes aspectos: análisis de la región codificante y las regiones limítrofes exón-intrón, aplicación de métodos para detectar variables puntuales y gruesas, usar la secuencia de referencia genómica correcta, evaluar los antecedentes e historia familiar del paciente y el tipo de mutación a determinar y evitar la aplicación paralela de técnicas para ahorrar costos. Es preferible aplicarlas de manera paulatina hasta encontrar la mutación.

Agradecimientos: Extendemos un sincero agradecimiento al Centro Gendiagnostik, principal patrocinador de este artículo de revisión, así como la SENACYT, que a través de su plataforma $\mathrm{ABC}$, desde la UNACHI, se permitió la descarga gratuita de numerosos artículos desde el portal ScienceDirect, haciendo la búsqueda bibliográfica más productiva.

\section{Referencias}

1. Gutmann DH, Aylsworth A, Carey JC, Korf B, Marks J, Pyeritz RE, MD, et al. The diagnostic evaluation and multidisciplinary management of neurofibromatosis 1 and neurofibromatosis 2. JAMA 1997; 278 (1): 51-7.

2. Huson SM. Neurofibromatosis: historical perspective, classification and diagnostic criteria. The Neurofibromatoses: A Pathogenic and Clinical Overview. Edited by SM Huson, RAC Hughes. London, Chapman \& Hall Medical, 1994. P. 1-22.

3. Jett K, Friedman JM. Clinical and genetic aspects of neurofibromatosis 1. Genet Med 2010; 12: 1-11.

4. NIH Consensus Development Conference. Neurofibro- 
matosis Conference Statement. Arch Neurol 1988; 45: 475-578.

5. Ferner RE, Huson SM, Thomas N, Moss C, Willshaw $\mathrm{H}$, Evans DG, et al. Guidelines for the diagnosis and management of individuals with neurofibromatosis 1 . J Med Genet 2007; 44: 81-8.

6. DeBella K, Szudek J, Friedman JM. Use of the national institutes of health criteria for diagnosis of neurofibromatosis 1 in children. Pediatrics 2000; 105(3): 608-14.

7. Cawthon R, Weiss R, Xu G, Viskochil D, Culver M, Stevens J, et al. A major segment of the neurofibromatosis type 1 gene: cDNA sequence, genomic structure, and point mutations. Cell 1990; 62: 193-201.

8. Wallace MR, Marchuk DA, Andersen LB, Letcher R, Odeh HM, Saulino AM, et al. Type 1 neurofibromatosis gene: identification of a large transcript disrupted in three NF1 patients. Science 1990; 249: 181-6.

9. Viskochil D, Buchberg AM, Xu G, Cawthon RM, Stevens J, Wolff RK, et al. Deletions and a translocation interrupt a cloned gene at the neurofibromatosis type 1 locus. Cell 1990; 62: 187-92.

10. Viskochil DH. Gene structure and function. Neurofibromatosis Type 1: From Genotype to Phenotype. Edited by M Upadhyaya, DN Cooper. Oxford, BIOS Publishers, 1998. p. 39-56.

11. Upadhyaya M. NF1 gene structure and NF1 genotype/ phenotype correlations. In: Neurofibromatoses. Edited by D Kaufmann. Basel, Karger, 2008. p. 46-62.

12. Cawthon RM, O'Connell P, Buchberg AM, Viskochil $\mathrm{D}$, Weiss $\mathrm{R}$, Culver M, et al. Identification and characterization of transcripts from the neurofibromatosis 1 region: the sequence and genomic structure of EVI2 and mapping of other transcripts. Genomics 1990; 7: 555-65.

13. Cawthon RM, Andersen LB, Buchberg AM, Xu GF, O'Connell P, Viskochil D, et al. cDNA sequence and genomic structure of Evi2B, a gene lying within an intron of the neurofibromatosis type 1 gene. Genomics 1991; 9: 446-60.

14. Viskochil D, Cawthon R, O’Connell P, Xu G, Stevens J, Culver $\mathrm{M}$, et al. The gene encoding the oligodendrocyte-myelin glycoprotein is embedded within the neurofibromatosis type 1 gene. Mol Cell Biol 1991; 11: 906-12.

15. Nishi T, Lee PSY, Oka K, Levin VA, Tanase S, Morino $\mathrm{Y}$, et al. Differential expression of two types of the neurofibromatosis type $1(\mathrm{NFl})$ gene transcripts related to neuronal differentiation. Oncogene 1991; 6: 1555-9.

16. Suzuki Y, Suzuki H, Kayama T, Yoshimoto T, Shibahara $\mathrm{S}$. Brain tumors predominantly express the neurofibromatosis type 1 gene transcripts containing the 63-base insert in the region coding for GTPase activating pro- tein-related domain. Biochem Biophys Res Commun 1991; 181: 955-61.

17. Ballester R, Marchuk D, Boguski M, Saulino A, Letcher $\mathrm{R}$, Wigler $\mathrm{M}$, et al. The NF1 locus encodes a protein functionally related to mammalian GAP and yeast IRA proteins. Cell 1990; 63: 851-9.

18. Fahsold R, Hoffmeyer S, Mischung C, Gille C, Ehlers C, Kucukceylan N, et al. Minor lesion mutational spectrum of the entire NF1 gene does not explain its high mutability but points to a functional domain upstream of the GAP-related domain. Am J Hum Genet 2000; 66: 790-818.

19. D’Angelo I, Welti S, Bonneau F , Scheffzek K. A novel bipartite phospholipid-binding module in the neurofibromatosis type 1 protein. EMBO Rep 2006; 7 (2): 174-9.

20. Martin GA, Viskochil D, Bollag G, McCabe PC, Crosier WJ, Haubruck H, et al. The GAP-related domain of the neurofibromatosis type 1 gene product interacts with ras p21. Cell 1990; 63: 843-9.

21. Xu GF, Lin B, Tanaka K, Dunn D, Wood D, Gesteland $\mathrm{R}$, et al. The catalytic domain of the neurofibromatosis type I gene product stimulates ras GTPase and complement IRA mutants of S. cerevisiae. Cell 1990; 63: 835-41.

22. Valero MC, Martín Y, Hernández-Imaz E, Marina Hernández A, Meleán G, Valero AM, et al. A highly sensitive genetic protocol to detect NF1 mutations. J Mol Diagn. 2011; 13 (2): 113-22.

23. Denayer E, de Ravel T, Legius E. Clinical and molecular aspects of RAS related disorders. J Med Genet 2008; 45 : 695-703.

24. Williams VC, Lucas J, Babcock MA, Gutmann DH, Korf B, Maria BL. Neurofibromatosis type 1 revisited. Pediatrics 2009; 123 (1): 124-33.

25. Guha A, Lau N, Huvar I, Gutmann D, Provias J, Pawson $\mathrm{T}$, et al. Ras-GTP levels are elevated in human NF1 peripheral nerve tumors. Oncogene 1996; 12: 507-13.

26. Jouhilahti E-M, Peltonen S, Heape AM, Peltonen J. The pathoetiology of neurofibromatosis 1. Am J Pathol 2011; 178 (5): 1932-9.

27. Novo Villaverde FJ. Efectos fenotípicos de las mutaciones. En: Martín-Romo M, Caicoya M, Editores, Genética Humana. Conceptos, mecanismos y aplicaciones de la Genética en el campo de la Biomedicina. Madrid, España. Pearson Educación, S.A.; 2007. p. 211-3.

28. Friedman JM. Neurofibromatosis 1.1998 oct 2 [updated 2014 sep 4]. In: Pagon RA, Adam MP, Ardinger HH, et al., editors. GeneReviews ${ }^{\circledR}$ [Internet]. Seattle (WA): University of Washington, Seattle; 1993-2014. http:// www.ncbi.nlm.nih.gov/books/NBK1109/

29. Brannan C, Perkins A, Vogel K, Ratner N, Nordlund M, 
Reid S, et al. Targeted disruption of the neurofibromatosis type-1 gene leads to developmental abnormalities in heart and various neural crest-derived tissues. Genes Dev 1994; 8: 1019-29.

30. Jacks T, Shih T, Schmitt E, Bronson R, Bernards A, Weinberg R. Tumour predisposition in mice heterozygous for a targeted mutation in Nf1. Nat Genet 1994; 7: 353-61.

31. Valero MC, Pascual-Castroviejo I, Velasco E, Moreno F, Hernández-Chico C. Identification of de novo deletions at the NF1 gene: no preferential paternal origin and phenotypic analysis of patients. Human Genetics 1997; 99 (6): 720-6.

32. Upadhyaya M, Cooper DN. The mutational spectrum in neurofibromatosis 1 and its underlying mechanisms. In: Upadhyaya M, Cooper DN (eds) Neurofibromatosis type 1: from genotype to phenotype. BIOS Scientific Publishers, Oxford; 1998. p. 65-88.

33. Jadayel D, Fain P, Upadhyaya M, Ponder M, Huson S, Carey J, et al. Paternal origin of new mutations in von Recklinghausen neurofibromatosis. Nature 1990; 343: 558-9.

34. Upadhyaya M, Ruggieri M, Maynard J, Osborn M, Hartog C, Mudd S, et al. Gross deletions of the neurofibromatosis type 1 (NF1) gene are predominantly of maternal origin and commonly associated with a learning disability, dysmorphic features and developmental delay. Hum Genet 1998; 102: 591-7.

35. De Raedt T, Brems H, López-Correa C, Vermeesch J, Marynen P, Legius E. Genomic organization and evolution of the NF1 microdeletion region. Genomics 2004; 84: 346-60.

36. Rasmussen SJ, Friedman JM. NF1 gene and neurofibromatosis 1. Am J Epidemiol 2000; 151: 33-40.

37. Ko JM, Sohn YB, Jeong SY, Kim HJ, Messiaen LM. Mutation spectrum of NF1 and clinical characteristics in 78 Korean patients with neurofibromatosis type 1 . Pediatr Neurol 2013; 48: 447-53.

38. Easton DF, Ponder MA, Huson SM, Ponder BAJ. An analysis of variation in expression of neurofibromatosis (NF) type 1 (NF1): evidence for modifying genes. Am J Hum Genet 1993; 53: 305-13.

39. Sabbagh A, Pasmant E, Laurendeau I, Parfait B, Barbarot $\mathrm{S}$, Guillot B, et al. Unravelling the genetic basis of variable clinical expression in neurofibromatosis 1 . Hum Mol Genet 2009; 18: 2768-78.

40. Upadhyaya M, Huson S, Davies M, Thomas N, Chuzhanova N, Giovannini S, et al. An absence of cutaneous neurofibromas associated with a 3-bp inframe deletion in exon 17 of the NF1 gene (c. 2970-2972 delAAT): evidence of a clinically significant NF1 genotype-phenotype correlation. Am J Hum Genet 2007; 80: 140-51.

41. Pasmant E, Sabbagh A, Spurlock G, Laurendeau I, Grillo E, Hamel MJ, et al. NF1 microdeletions in neurofibromatosis type 1: from genotype to phenotype. Hum Mutat 2010; 31 (6): E1506-18.

42. Mautner VF, Kluwe L, Friedrich RE, Roehl AC, Bammert S, Högel J, et al. Clinical characterization of 29 neurofibromatosis type- 1 patients with molecularly ascertained 1.4 Mb type-1 NF1 deletions. J Med Genet 2010; 47 (9): 623-30.

43. Trovó AB, Goloni-Bertollo EM, Tajara EH. Neurofibromatose tipo 1: uma revisao. HB Cientifica 2002; 9: 98-110.

44. Ferner R: The neurofibromatoses. Pract Neurol 2010; 10: 82-93.

45. Rodillo E, Fruns M, Ferrada MJ, Demarta JC, Adlerstein L. Neurofibromatosis tipo I: una enfermedad de manifestaciones heterogéneas. Rev Chil Pediatr 1996; 67 (1): 1-5.

46. Zoller M, Rembeck B, Akesson HO, Angervall L. Life expectancy, mortality and prognostic factors in neurofibromatosis type 1. A twelve-year follow-up of an epidemiological study in Goteborg, Sweden. Acta Derm Venereol 1995; 75: 136-40.

47. Evans DG, Baser ME, McGaughran J, Sharif S, Howard E, Morán A. Malignant peripheral nerve sheath tumours in neurofibromatosis 1. J Med Genet 2002; 39: 311-4.

48. Ferner RE, Gutmann DH. International consensus statement on malignant peripheral nerve sheath tumors in neurofibromatosis 1. Cancer Res 2002; 62: 1573-7.

49. Ruggieri M, Huson SM. The clinical and diagnostic implications of mosaicism in the neurofibromatoses. Neurology 2001; 56: 1433-43.

50. Boyd K, Korf B, Theos A. Neurofibromatosis type 1. J Am Acad Dermatol 2009; 61 (1): 1-16.

51. De Raedt T, Maertens O, Serra E, Legius E. Somatic NF1 mutations in tumors and other tissue. In: Neurofibromatoses. Edited by D Kaufmann. Basel, Karger, 2008, pp 143-53.

52. Whitehouse D. Diagnostic value of the café-au-lait spot in children. Arch Dis Child 1966; 41: 316-9.

53. McKeever K, Sheperd CW, Crawford H, Morrison PJ. An epidemiological, clinical and genetic survey of Neurofibromatosis type 1 in children under sixteen years of age. Ulster Med J 2008; 77 (3): 160-3.

54. Messiaen L, Wimmer K. NF1 mutational spectrum. In: Neurofibromatoses. Edited by D Kaufmann. Basel, Karger, 2008. p 63-77.

55. Messiaen L, Callens T, Mortier G, Beysen D, Vandenbroucke I, Van Roy N, et al. Exhaustive mutation analysis of the NF1 gene allows identification of $95 \%$ 
of mutations and reveals a high frequency of unusual splicing defects. Hum Mutat 2000; 15: 541-55.

56. Verlinsky Y, Rechitsky S, Verlinsky O, Chistokhina A, Saharapova T, Masciangelo C, et al. Preimplantation diagnosis for neurofibromatosis. Reprod Biomed Online 2002; 4: 218-22.

57. Huson SM. The neurofibromatosis: classification, clinical features and genetic counselling. In: Neurofibromatoses. Edited by D Kaufmann. Basel, Karger, 2008. p. $1-20$.

58. Brems H, Chmara M, Sahbatou M, Denayer E, Taniguchi K, Kato R, et al. Germline loss-of-function mutations in SPRED1 cause a neurofibromatosis 1-like phenotype. Nat Genet 2007; 39: 1120-6.

59. Messiaen L, Yao S, Brems H, Callens T, Sathienkijkanchai A, Denayer E, et al. Clinical and mutational spectrum of neurofibromatosis type 1-like syndrome. JAMA 2009; 302: 2111-8.

60. De Luca A, Schirinzi A, Buccino A, Bottillo I, Sinibaldi L, Torrente I, et al. Novel and Recurrent Mutations in the NF1 Gene in Italian Patients with Neurofibromatosis Type 1. Human mutation. Mutation in Brief \#716 (2004) Online. DOI:10.1002/humu.9245.

61. Ars E, Kruyer H, Morell M, Pros E, Serra E, Ravella A, et al. Recurrent mutations in the NF1 gene are common among neurofibromatosis type 1 patients. Online mutation report. J Med Genet 2003; 40: e82.

62. Shen MH, Harper PS, Upadhyaya M. Molecular genetics of neurofibromatosis type 1(NF1). Review article. J Med Genet 1996; 33: 2-17.

63. Korf BR. The NF1 genetic analysis consortium. In: Upadhyaya M, Cooper DN (eds) Neurofibromatosis type 1: from genotype to phenotype. BIOS Scientific Publishers, Oxford; 1998. p. 57-63.

64. Trovó AB, Goloni-Bertollo EM, Mancini UM, Rahal P, De Azevedo Jr. WF, Tajara EH. Mutational analysis of the GAP-related domain of the neurofibromatosis type 1 gene in Brazilian NF1 patients. Genetics and Molecular Biology 2004; 27 (3): 326-30.

65. De Luca A, Buccino A, Gianni D, Mangino M, Giustini $S$, Richetta A, et al. NF1 gene analysis based on DHPLC. Hum Mutat 2003; 21: 171-2 (http://www.jmedgenet. $\mathrm{com} /$ cgi/content/full/40/6/e82).

66. De Luca A, Bottillo I, Dasdia MC, Morella A, Lanari V, Bernardini L, et al. Deletions of NF1 gene and exons detected by multiplex ligation dependent probe amplification. J Med Genet 2007; 44: 800-8.

67. Orraca Castillo M, Lantigua Cruz A, Licourt Otero D. Cariotipo de alta resolución en sangre periférica en la Neurofibromatosis 1. Sociedades científicas. 2009_

68. Novo Villaverde FJ. Diagnóstico de enfermedades genéticas. En: Martín-Romo M, Caicoya M, Editores, Genética Humana. Conceptos, mecanismos y aplicaciones de la Genética en el campo de la Biomedicina. Madrid, España. Pearson Educación, S.A.; 2007. p. 228-31.

69. Weihong $\mathrm{Xu}$, Xiao Yang, Xiaoxia Hu, Shibo Li. Fifty-four novel mutations in the NF1 gene and integrated analyses of the mutations that modulate splicing. International journal of molecular medicine 2014; 34 : 53-60.

70. Kluwe L, Siebert R, Gesk S, Friedrich RE, Tinschert S, Kehrer-Sawatzki H, et al. Screening 500 unselected neurofibromatosis 1 patients for deletions of the NF1 gene. Hum Mutat 2004; 23: 111-6. 